This document is the Accepted Manuscript version of a Published Work that appeared in final form in Journal of the American Chemical Society, copyright (c) American Chemical Society after peer review and technical editing by the publisher.

To access the final edited and published work see: https://doi.org/10.1021/jacs.6b03710 


\title{
A Surface cis Effect: Influence of an Axial Ligand on Molecular Self-Assembly
}

\author{
Thomas Knaak, ${ }^{\dagger}$ Thiruvancheril G. Gopakumar, ${ }^{\ddagger}$ Bettina Schwager, $₫$ Felix \\ Tuczek, Roberto Robles, ${ }^{\S}$ Nicolás Lorente,\| and Richard Berndt*,† \\ $\dagger$ Institut für Experimentelle und Angewandte Physik, Christian-Albrechts-Universität zu \\ Kiel, Germany \\ $\ddagger$ Department of Chemistry, Indian Institute of Technology Kanpur, Kanpur 208016, India \\ \Institut für Anorganische Chemie, Christian-Albrechts-Universität zu Kiel, Germany \\ $\S$ Catalan Institute of Nanoscience and Nanotechnology (ICN2), CSIC and The Barcelona \\ Institute of Science and Technology, Campus UAB, Bellaterra, 08193 Barcelona, Spain \\ $\|$ Centro de Física de Materiales CFM/MPC (CSIC-UPV/EHU), Paseo Manuel de \\ Lardizabal 5, 20018 Donostia-San Sebastián, Spain \\ 83 Donostia International Physics Center (DIPC), Paseo Manuel de Lardizabal 4, 20018 \\ Donostia-San Sebastián, Spain \\ E-mail: berndt@physik.uni-kiel.de
}




\begin{abstract}
Adding ligands to molecules can have drastic and unforeseen consequences in the final products of a reaction. Recently a surface trans effect due to the weakening of a molecule-surface bond was reported. Here, we show a surface cis effect where an axial ligand at adsorbed transition-metal complexes enables lateral bonding among the molecules. In the absence of this ligand, the intermolecular interaction is repulsive and supramolecular patterns are not observed. Fe-tetramethyl-tetraazaannulene on $\mathrm{Au}(111)$ was investigated using low-temperature scanning tunneling microscopy and spectroscopy along with density functional theory calculations. At low coverages the molecules remain isolated. Exposure to CO leads to axial CO bonding and induces reordering into extended clusters of chiral molecular trimers. The changed self-assembly pattern is due to a CO-induced modification of the molecular structure and the corresponding charge transfer between the molecule and the substrate, which in turn changes the lateral intermolecular forces.
\end{abstract}




\section{Introduction}

The properties of metalorganic complexes may be controlled through the number and type of ligands attached to the metal center. This concept from solution chemistry ${ }^{1-5}$ has been extended to complexes adsorbed on metal surfaces. Both electronic and magnetic properties have been shown to respond to the addition or removal of a ligand. ${ }^{6-20}$

Little is known about the influence of axial ligands on intermolecular interactions at surfaces. For planar metal complexes on surfaces, the ligands naturally occupy the axial position on the metal atom of the complex, conferring the metal atom with an octahedral-like ligand field, which changes the otherwise square-planar ligand field with consequences regarding the electronic and magnetic properties of the complex. Phthalocyanines, porphyrins and similar molecules belong to this class of metallorganic complexes with promise for ligand-induced modifications. $^{21-25}$

The trans effect in coordination chemistry is the weakening of a ligand on the metal center by the attachment of an additional ligand at a trans position. A closely related surface trans effect has recently been reported from NO bonding to metal tetraphenyl porphyrins on $\mathrm{Ag}(111) .{ }^{18}$ An overview of the surface trans effect and the closely related surface spin trans effect is available in a recent review. ${ }^{24}$ As to intermolecular interactions, coadsorption of NO and largish molecules ${ }^{26-31}$ has been reported to affect the molecular arrangements on a surface. In these cases the transition-metal complex and the second species was co-adsorbed side-by-side on the metal substrate and did not bind to the metal center as an additional ligand.

Here, we report on the formation of supramolecular bonds on a surface induced by the addition of an axial ligand to the metal center of a complex. We reveal this effect using CO ligands on Fe-tetramethyl-tetraazaannulene (FeTMTAA, Fig. 1a, b) on Au(111). While pristine FeTMTAA molecules on Au maximize their mutual distances at low coverages, the attachment of $\mathrm{CO}$ leads to a drastic rearrangement. CO causes the molecules to aggregate into ordered clusters. We discuss this striking observation in terms of the structure of 
the molecule, electrostatic intermolecular interactions and the effect of $\mathrm{CO}$ on the charge transfer between FeTMTAA and the substrate. Moreover, we compare the results with equivalent measurements on Ni-tetramethyl-tetraazaannulene (NiTMTAA) ${ }^{32}$ and density functional theory (DFT) calculations. It turns out that CO attaches axially to FeTMTAA, which lies flat on the substrate. As a result the interaction of the complex with its neighbors is changed from repulsive to attractive, which may be viewed as a surface cis effect.

\section{Methodology}

\section{Experiment}

Experiments were performed with a home-built STM operated in ultrahigh vacuum (UHV) at $5 \mathrm{~K}$ and $11 \mathrm{~K}$. FeTMTAA was synthesized according to Ref. 33 and deposited onto clean $\mathrm{Au}(111)$ surfaces at room temperature by sublimation from a Ta crucible. Prior to sublimation the material was repeatedly degassed close to its sublimation temperature of $\approx 200^{\circ} \mathrm{C}$ in $\mathrm{UHV}$. Exposure to $\mathrm{CO}$ was performed at $300 \mathrm{~K}$ and a $\mathrm{CO}$ pressure of $\approx$ $4 \cdot 10^{-5}$ mbar for 30 to 40 minutes. Prepared samples were transferred to the cold STM. Tips were electrochemically etched from tungsten wire and further prepared in situ by indentation into the substrate. A sinusoidal modulation $\left(10 \mathrm{mV}_{r m s}, 7 \mathrm{kHz}\right)$ was added to the sample voltage $V$ to record spectra of the differential conductance.

\section{Theory}

We applied DFT as implemented in the VASP $\operatorname{code}^{34}$ using the generalized gradient approximation proposed by Perdew, Burke, and Ernzerhof $(\mathrm{PBE})^{35}$ to treat electronic exchange and correlation. Dispersion-corrections are included through the scheme proposed by Tkatchenko and Scheffler. ${ }^{36}$ We have used a plane wave basis set and the projected augmented wave (PAW) method $^{37}$ implemented in VASP with an energy cut-off of $280 \mathrm{eV}$. The surface was represented by a five-layer slab and in all calculations we allowed the relaxation of the sub- 
strate atoms in the two top-most metal layers as well as all the atoms of the adsorbates. The substrate atoms in the three bottom layers were kept fixed to their bulk equilibrium positions. All geometry optimizations were carried out until the forces on mobile atoms were smaller than $0.02 \mathrm{eV} / \AA$. The molecules were arranged in a periodic pattern formed by lozenges of $25 \mathrm{Au}$ atoms following the ideal surface of $\mathrm{Au}(111)$. The $k$-point sampling corresponds to a Monkhorst and Pack mesh of $3 \times 3 \times 1$. DFT is known to underestimate the exchange splitting of localized orbitals, leading to an underestimation of magnetic moments. In order to correct for this we have included an intra-atomic correlation correction $U-J=3$ $\mathrm{eV}$, where $U$ corresponds to the Coulomb interaction and $J$ to the exchange coupling, using the scheme by Dudarev et al. ${ }^{38}$

\section{Results}

\section{FeTMTAA adsorption on Au(111)}

The macrocyclic ligand of FeTMTAA exhibits a pronounced saddle shape (Figs. 1a and b) owing to the steric interaction of the four methyl groups with the phenyl rings. ${ }^{39}$ Figure $1 \mathrm{~b}$ shows a STM topograph of 0.2 monolayers (ML) FeTMTAA on Au(111). ${ }^{40}$ Single molecules are clearly resolved as oval protrusions. Some examples are indicated by elongated hexagons in Fig. 1. The molecules form a fairly regular hexagonal pattern with an intermolecular distance of $\approx 1.9 \mathrm{~nm}$. Molecules are aligned with their long axis along one of the three compact directions of $\mathrm{Au}(111)$. The reconstruction of the $\mathrm{Au}$ substrate causes small height variations. ${ }^{41,42}$ In particular, the transition region between fcc and hcp stacked areas of Au is elevated by $\approx 15 \pm 4 \mathrm{pm}$ and this height difference is also found on the molecules $(\approx 20 \mathrm{pm}$ in our data).

Intramolecular contrast is demonstrated in Fig. 2a, which reveals that the molecules appear higher along the short axis. The STM image therefore indicates that FeTMTAA adsorbs with its phenyl rings pointing toward the substrate. While this geometric interpretation of 
the image contrast is a simplification, it is important to note that the STM image of FeTMTAA varies little over a range of sample voltages. This observation is consistent with spectra of the differential conductance $(d I / d V)$ acquired above the center of FeTMTAA, which do not exhibit specific molecular states over a bias range of $3 \mathrm{~V}$ (Fig. 3).

Our calculations corroborate the above findings. An energy minimum was found for the molecule horizontally adsorbed on the surface with the methyl groups pointing into vacuum. This enables a strong bonding of the Fe center to the substrate. There is a weak preference for placing the Fe atom on top of a surface Au atom, with a chemisorption energy of $-2.35 \mathrm{eV}$ $(-54.2 \mathrm{kcal} / \mathrm{mol})$. Adsorption at the fcc hollow and at bridge sites are less favorable by 80 and $800 \mathrm{meV}$, respectively. The small energy difference between the fcc and top adsorption sites suggests that molecules may be found to occupy different sites. The van der Waals interaction leads to a considerably flattening of the adsorbed molecule reducing the saddleshape of the gas-phase molecule as can be seen in the calculated geometrical structure of Fig. 4.

The optimized geometry of the molecule is actually affected by the molecular magnetic moment. On the top site we find that the FeTMTAA molecule presents two possible spin values - high-spin $4.3 \mu_{B}$, low-spin $2.6 \mu_{B}$ - with an energy difference of $23 \mathrm{meV}$. In the highspin configuration the Fe ion is $2.7 \AA$ away from the $\mathrm{Au}(111)$ surface, while the entire molecule is lifted by approximately $0.1 \AA$ for the low-spin configuration. The hollow-site configuration, which is very close in energy (20 meV higher than the top-site configuration), pushes the Fe ion further to $2.9 \AA$. The charge transfer involving the Fe ion confers the molecule with its main properties. Indeed, this is corroborated by a more profound inspection of the STM images of FeTMTAA that reveals a central protrusion.

A Bader charge analysis of the adsorbed system reveals a net electron donation from the molecule to the substrate. The Fe atom loses 0.2 electrons whereas the $\mathrm{N}$ atoms acquire 0.08 electrons. The C-atoms remain largely unaffected. Figure 4 displays the differences of electronic density due to the formation of the molecule-substrate bond. This induced charge 
is computed using the expression:

$$
\delta \rho=\rho_{\text {all }}-\rho_{\text {molecule }}-\rho_{\text {substrate }}
$$

where $\rho_{\text {all }}$ is the density of electrons of the full system, $\rho_{\text {molecule }}$ is the density of electrons for the molecule in exactly the same geometry as the adsorbed molecule and $\rho_{\text {substrate }}$ is the corresponding density of the surface also with the adsorption geometry.

In the experiments, most of the isolated FeTMTAA molecules could only be stably imaged at low bias $|V|<0.2 \mathrm{~V}$. When the voltage exceeded this values the molecules moved and the tunneling current became unstable. This fact agrees with the picture emerging from the calculations. The adsorbed molecules have acquired a positive charge and therefore interact with the strong and inhomogeneous electrical field under the STM tip. The charge analysis together with the computed energy landscape of the molecule on the surface provide a distinct picture of the molecular arrangement on the surface. Namely, the positive charge along with the corresponding image charge lead to repulsion via dipole-dipole interactions on a potential energy surface with little corrugation. The result, in equilibrium, is an array of equidistant single molecules.

A projection of the density of states (PDOS) on the $d$-orbitals of the Fe ion provides further information on the electronic and magnetic properties of the molecule. Figure 5 shows the PDOS for the free molecule as well as for the high- and low-spin configurations of the adsorbed molecule. Compared to the PDOS of the free molecule (black and dashed) the minority-spin components indeed carry less charge for the adsorbed species, in agreement with the donation of charge from the molecule. For the low-spin case, Fig. $5(b)$, we see that charge transfer mainly affects the minority spin, reducing the magnetic moment of the molecule. Nevertheless, the low-spin molecule approximately maintains the magnetic moment of the free molecule, corresponding to a total spin $S=1$. In the high-spin case, the donation from the minority channel is partially compensated from back-donation into 
the majority-spin $d$-states, which explains the larger magnetic moment of this configuration. The high-spin molecule rather corresponds to a total spin $S=2$.

The electronic systems described above may be described as approximate $d^{6}$ configurations of the Fe ion, where only the actual filling of the different $d$-shells change. In the case of the free molecule, the Fe ion is in a $d^{6}$ configuration, leading to $S=1$ and to a Fe(II) valence. Both high- and low-spin states involve a small reduction of the $d$-shell occupation, well below the transfer of one electron, and hence they remain in an approximate $d^{6}$ configuration corresponding to an Fe(II) oxidation state. The spin configurations that we find ( $S=2$ and $S=1$ ) are indeed compatible with the Fe(II) oxidation state. ${ }^{43}$

\section{Adsorption of CO on FeTMTAA on $\mathrm{Au}(111)$}

FeTMTAA-covered surfaces were exposed to $\mathrm{CO}$ at ambient temperature. Because CO desorbs from gold below ambient temperature ${ }^{44}$ no CO remains on the Au substrate. Subsequent imaging at low temperature revealed drastic changes. Figure 1c was recorded at 0.2 ML coverage and may be directly compared to Fig. 1b with the same coverage but without CO. FeTMTAA molecules in fcc areas are no longer isolated but have aggregated into small supramolecular assemblies the main building block being a chiral trimer. ${ }^{45-48}$ In addition, the intramolecular contrast is modified (Fig. 2b). The CO-exposed molecules (denoted CO-FeTMTAA below) exhibit a depression at their center. The images of CO-FeTMTAA are voltage dependent in contrast to those of FeTMTAA. The conductance spectrum acquired at the center of the molecules reveals states at -0.9 and $-1.3 \mathrm{~V}$, which are close to those of NiTMTAA (Fig. 3).

The observed CO-induced changes in STM images and $d I / d V$ spectra strongly suggest that $\mathrm{CO}$ is binding to FeTMTAA on $\mathrm{Au}(111)$. Below, we argue that $\mathrm{CO}$ attaches axially to the Fe center on the vacuum (rather than substrate) side of the molecule. The additional ligand apparently modifies the intermolecular interaction from repulsive in the FeTMTAA case to attractive for CO-FeTMTAA. 
The influence of the $\mathrm{CO}$ ligand on the intermolecular interactions is also obvious on a larger scale and at higher coverages. Figures 2c-f present images of FeTMTAA and COFeTMTAA, each at 0.5 ML coverage. The favorable fcc areas are almost entirely covered with molecules. Pristine FeTMTAA forms double rows with neighboring molecules rotated by $60^{\circ}$ with respect to each other (Fig. 2c and e). CO-FeTMTAA, by contrast, forms zigzag chains (Fig. 2d and f), whose building blocks again are trimers (Fig. 2b). The zigzag chains are in fact porous, honeycomb-like networks, ${ }^{49}$ which closely resemble the pattern observed from NiTMTAA ${ }^{32}$ (Fig. 1d).

Our DFT calculations for CO-FeTMTAA in gas phase led to an optimized geometry where CO binds axially to FeTMTAA (Fig. 1c). CO-FeTMTAA being a closed-shell system, this result is reproduced in calculations for CO-FeTMTAA on the inert $\mathrm{Au}(111)$ surface. Moreover, an axial coordination of CO to FeTMTAA in crystals was previously found with X-ray diffraction. ${ }^{50} \mathrm{~A}$ slight displacement of the $\mathrm{Fe}$ atom from the plane of the $\mathrm{N}$ atoms leads to nonequivalent axial ligand sites and a preference for coordination at the side of the phenyl benzenoid rings. ${ }^{39,51}$ Because of the orientation of FeTMTAA on $\mathrm{Au}(111)$, however, this site is located at the substrate side of the molecule that is not favorable energetically due to the steric hindrance between surface and molecule.

Carbon monoxide easily binds to the free FeTMTAA molecule. As for the heme group of porphyrin molecules, the adsorption of $\mathrm{CO}$ is made possible by a charge transfer into the $2 \pi$ orbital of carbon monoxide. The adsorption of $\mathrm{CO}$ on FeTMTAA molecules is qualitatively similar to the adsorption of $\mathrm{CO}$ on porphyrins although the amount of charge transfer to the $\mathrm{CO}$ molecule is larger signaling more chemical activity from the FeTMTAA molecule than from porphyrins. ${ }^{52}$

When the combined CO-FeTMTAA molecule is adsorbed on $\mathrm{Au}(111)$ no charge transfer from $\mathrm{Fe}$ to the $\mathrm{Au}$ substrate takes place, in contrast to the case of pristine FeTMTAA. This is clearly seen in the almost identical Bader charge distributions before and after COFeTMTAA adsorption on $\mathrm{Au}(111)$. Figure $6(a)$ shows the value of the induced electron 
density, Eq. (1) for the same isovalue as Fig. 4. The charge is very reduced, showing the small transfer of charge between molecular complex and surface. Figure $6(b)$ is the induced charge averaged over planes parallel to the surface in the unit cell of the calculation. The Fe atom loses up to 5 electrons per $\mathrm{nm}^{3}$ due to the adsorption of FeTMTAA on Au (111), while this value is reduced to 2 electrons per $\mathrm{nm}^{3}$ when CO-FeTMTAA is considered. This leads to a smaller overall induced dipole when $\mathrm{CO}$ is adsorbed on FeTMTAA. From these results, we conclude that while FeTMTAA becomes positively charged on the $\mathrm{Au}(111)$ surface, COFeTMTAA is largely neutral. This is further corroborated by the experimental observation that isolated CO-FeTMTAA molecules on the surface were stable at elevated sample voltages. Otherwise, the electrical field from the tip would have likely influenced the molecule.

The magnetic moment of the new system is zero. This is due to the rearrangement of the $d$-shell of the $\mathrm{Fe}(\mathrm{II})$ ion. Indeed, the valency $\mathrm{Fe}(\mathrm{II})$ is compatible ${ }^{43}$ with molecular spins of 0,1 and 2 , as we have found here.

Adsorption to the fcc site is $110 \mathrm{meV}$ more stable than the bridge configuration and $1.7 \mathrm{eV}$ more stable than the top site adsorption. The adsorption of CO-FeTMTAA is qualitatively different from the case of FeTMTAA. This is due to the partial passivation of the Fe center when $\mathrm{CO}$ is directly attached to it. The adsorption of CO-FeTMTAA is directed by the interaction of the nitrogen atoms with the substrate. The hollow site enables a close interaction of two nitrogen atoms with two gold atoms, stabilizing the molecule. In the absence of $\mathrm{CO}$, the Fe ion binding activity increases, changing the adsorption site preference. Nevertheless, the chemisorption energies of CO-FeTMTAA $(-2.83 \mathrm{eV})$ and FeTMTAA $(-2.35 \mathrm{eV})$ are of similar magnitude despite the qualitative differences in the bonding.

The diminished reactivity of the Fe ion leads to an increased Fe-surface distance. For the fcc adsorption site, this distance is $3.5 \AA$, i. e. $0.6 \AA$ more than for the FeTMTAA molecule. At first glance it may seem surprising that the CO-FeTMTAA molecule appears lower than FeTMTAA in STM images because some small ligands at transition metal complexes were previously imaged as protrusions. ${ }^{8,17,19}$ However, from our calculations we find that the low 
apparent height of CO-FeTMTAA is due to an electronic effect. A distant metallic tip will image the nodal plane of the CO $2 \pi^{*}$ orbital as a depression. This is similar to CO on $\mathrm{Cu}(111)$ where the molecule reduces the conductance and leads to a depression in constantcurrent STM images over a range of bias voltages. ${ }^{53,54}$ Similarly, bonding of $\mathrm{H}$ to the Mn center of MnPc was observed to cause a depression in experimental and calculated STM images. ${ }^{55}$

\section{Discussion}

The addition of a small molecule as an axial ligand to an adsorbed molecule can destabilize the molecular bond to the surface. Hieringer et al. demonstrated this surface trans effect for metal-tetraphenylporphyrins and their nitrosyl complexes on a $\operatorname{Ag}(111)$ surface. ${ }^{18}$ Our observations of CO-induced reorganization of FeTMTAA show that the addition or removal of an axial ligand may additionally modify the interactions with nearest-neighbor molecules in the surface plane. As these molecules are necessarily located in a cis position relative to their CO ligands this change may be viewed as a new form of cis effect that occurs on surfaces.

A priori, several factors can be at the origin of the surface cis effect. The $\mathrm{Au}(111)$ substrate with its herringbone reconstruction may play a role as well as the electronic surface state, which is known to mediate an oscillatory long-range interaction. ${ }^{56-62}$ None of these factors, however, is significantly different for FeTMTAA and NiTMTAA, in contrast to the patterns they form. Consequently, the differences between FeTMTAA and NiTMTAA and the similarities between CO-FeTMTAA and NiTMTAA are linked to the molecules themselves and their bonds to the substrate.

The effect of $\mathrm{CO}$ is threefold as deduced from the above experimental and theoretical analysis. First, CO changes the geometrical structure of the FeTMTAA molecule. According to our DFT calculations of free molecules, the distance between the outermost $\mathrm{C}$ atoms 
along the long axis of the FeTMTAA molecule (Fig. 1a) (963 pm and $971 \mathrm{pm}$ in spin 0 and 1 states, respectively) is reduced in CO-FeTMTAA (950 pm). The latter value is identical to the distance calculated for NiTMTAA. In other words, the reaction with CO geometrically converts FeTMTAA to NiTMTAA. The same trend is calculated for the adsorbed molecules. Second, a similar conversion is found at the electronic level, where we see that the adsorbed NiTMTAA presents the same features as the adsorbed CO-FeTMTAA (see Supporting Information). In agreement with the calculated results, the experimental spectra of CO-FeTMTAA and NiTMTAA exhibit nearly identical peaks. Finally, bonding of CO to the $\mathrm{Fe}$ center weakens the $\mathrm{Fe}-\mathrm{Au}$ bond and thus diminishes the charge transfer. As a result, the electrostatic interaction between FeTMTAA molecules is affected. FeTMTAA is positively charged while CO-FeTMTAA is not leading to net repulsion among FeTMTAA molecules that is absent for CO-FeTMTAA. This affects the actual molecular arrangement. FeTMTAA molecules mutually repel owing to their charge state and tend to form a Wigner crystal. CO-FeTMTAA molecules on the other hand tend to form clusters. Their charge state is modified, the electrostatic interaction is reduced, and consequently their arrangement is dictated by the threefold symmetry of the substrate, the intermolecular van der Waals attraction and the steric forces of the ligand groups. The similarity of the CO-FeTMTAA patterns with those of NiTMTAA indicates that CO substantially reduces the electrostatic repulsion, which is consistent with the results of our DFT calculations.

At large coverages FeTMTAA molecules in fcc areas aggregate. A related observation was reported from the molecular donor tetrathiafulvalene on $\mathrm{Au}(111){ }^{63,64}$ While it forms a Wigner crystal at low coverage its charge transfer to the surface is reduced at higher coverages and aggregation occurs. Reduced coupling at high coverage was also found for $\mathrm{FePc}$ on $\mathrm{Ag}(111) \cdot{ }^{65}$ 


\section{Conclusions}

In summary, low-temperature STM data from FeTMTAA on Au(111) show that exposure to CO drastically changes the molecular pattern. A combined experimental and theoretical study shows that the CO coordination to the Fe center leads to a modified binding to the substrate. Beyond weakening the Fe-substrate bond, which is typical of a trans effect, the CO ligand modifies the lateral interaction between molecules. While the trans effect relies on orbital-mediated interaction of ligands, the surface cis effect reported here involves a ligand-induced charge redistribution between the molecule and its substrate. The presence of $\mathrm{CO}$ axial ligands permits the creation of a supramolecular structure while the absence of the ligands leads to the repulsion of the FeTMTAA molecules on $\mathrm{Au}(111)$.

\section{Acknowledgement}

Funding by the Deutsche Forschungsgemeinschaft through Sonderforschungsbereich 677 is gratefully acknowledged. ICN2 acknowledges support from the Severo Ochoa Program (MINECO, Grant SEV-2013-0295). Financial support from MINECO MAT2015-66888-C32-R is also gratefully acknowledged.

\section{Supporting Information Available}

(1) Model structures of FeTMTAA and CO-FeTMTAA chains. (2) STM image of complete monolayer of FeTMTAA. (3) Density of states of CO-FeTMTAA. (4) Electronic structure of NiTMTAA. This material is available free of charge via the Internet at http://pubs . acs. org/. 


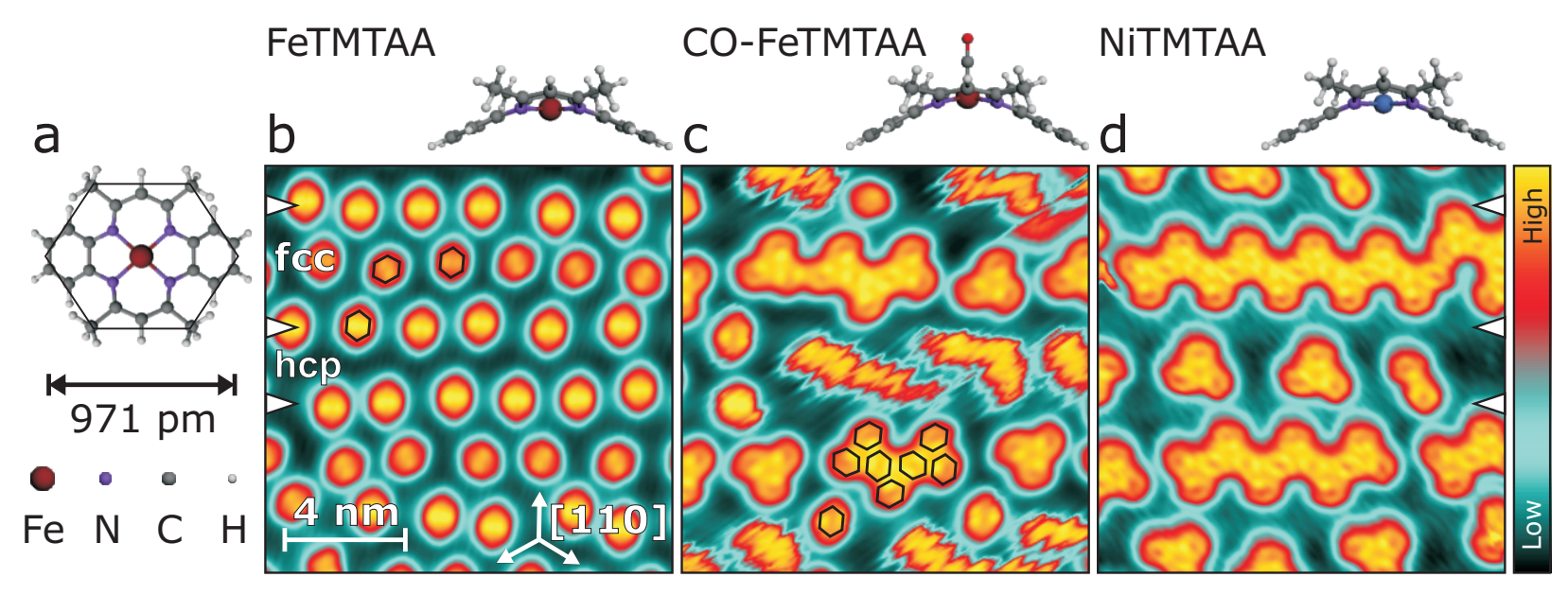

Figure 1: (a) Top view of the calculated gas-phase structure of FeTMTAA. Dark red, violet, dark grey, and light grey spheres indicate Fe, N, C, and H atoms, respectively. The model resembles an elongated hexagon (line), which is used to indicate the molecules and their orientations in STM images. (b) STM topograph $(I=50 \mathrm{pA}, V=0.1 \mathrm{~V})$ of $0.2 \mathrm{ML}$ of FeTMTAA on $\mathrm{Au}(111)$ along with a side view of the gas-phase structure of FeTMTAA. (c) The same coverage of FeTMTAA imaged after exposure to CO. $I=50 \mathrm{pA}, V=0.2 \mathrm{~V}$. A side view of the structure of CO-FeTMTAA is also shown (light red indicating $\mathrm{O}$ ). The molecular pattern has drastically changed with many molecules assembled into trimers and chains. Monomers in hcp areas appear fuzzy because they move during scanning. At low voltages, many monomers are more stable and are identified as pristine FeTMTAA molecules. (d) $0.2 \mathrm{ML}$ of NiTMTAA. $I=100 \mathrm{pA}, V=0.5 \mathrm{~V}$. The pattern is closely related to that of CO-FeTMTAA. A side view of the calculated structure is shown for each molecule. 


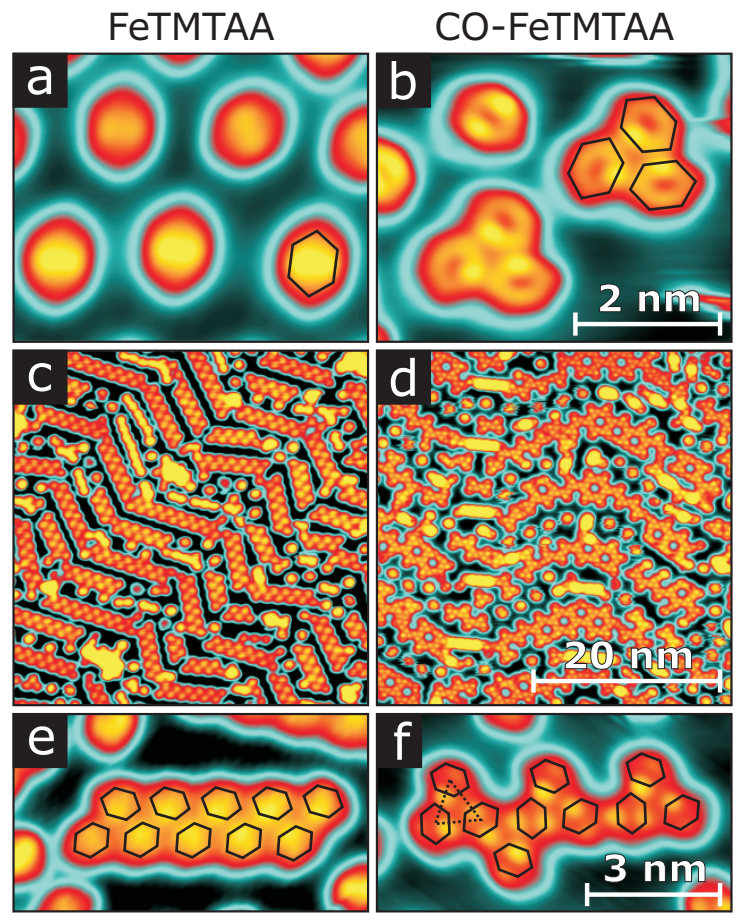

Figure 2: STM topographs of FeTMTAA and CO-FeTMTAA on Au(111). (a, b) Detailed images of $0.2 \mathrm{ML}$. (c, d) $0.5 \mathrm{ML}$. (e, f) Detailed images of chains of FeTMTAA and CO-FeTMTAA at $0.5 \mathrm{ML}$ coverage. The color palette from Fig. 1 is used, with some contrast enhancement in $\mathrm{c}$ and $\mathrm{d}$. Tunneling parameters: $I=50 \mathrm{pA}$ and $V=0.1,0.2,-0.25,-0.5,-0.06$, and $-0.5 \mathrm{~V}$ in $(\mathrm{a}-\mathrm{f})$, respectively. 


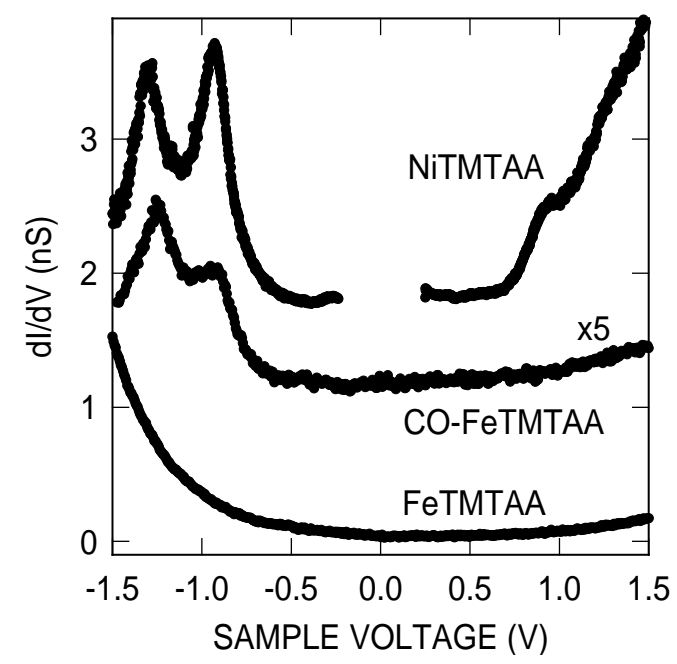

Figure 3: $d I / d V$ spectra acquired above the centers of FeTMTAA, CO-FeTMTAA, and NiTMTAA molecules recorded at coverages of $0.8,0.5$, and $0.5 \mathrm{ML}$, respectively. Current feedback was opened at $V=1.5 \mathrm{~V}, I=100 \mathrm{pA}$ and $V=-1.5 \mathrm{~V}, I=200 \mathrm{pA}$ for FeTMTAA and CO-FeTMTAA, respectively. The NiTMTAA data were recorded at a constant current of $I=1 \mathrm{nA}$ and converted to an approximate constant-height spectrum using the procedure of Ref. ${ }^{66}$ As the tip-sample distance is unknown, the calculated conductance is arbitrarily scaled for NiTMTAA. The CO-FeTMTAA data were multiplied by a factor of 5 . The COFeTMTAA and NiTMTAA spectra have been shifted by 1 and $1.5 \mathrm{nS}$, respectively. 

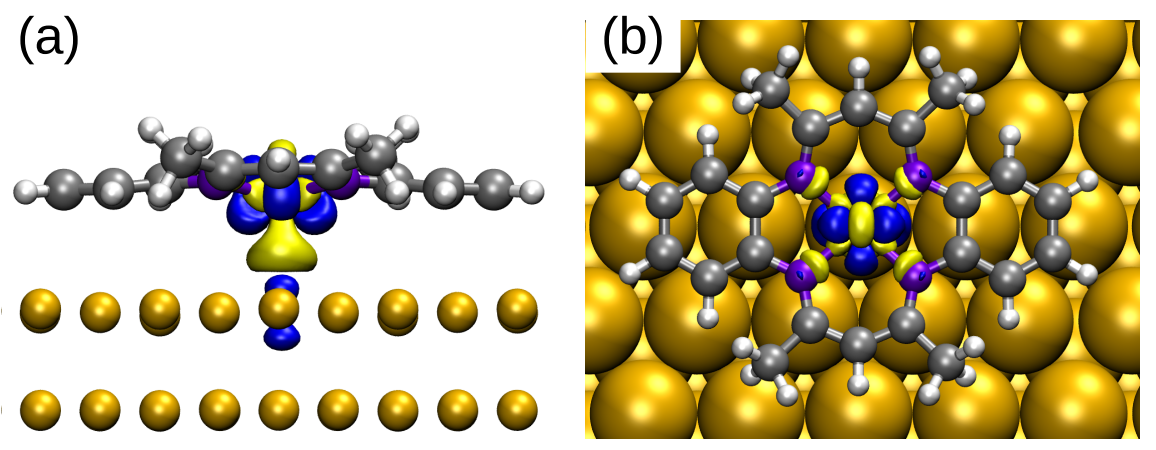

Figure 4: Electronic density induced by the molecule-substrate interaction. The isosurfaces correspond to a value of $\pm 0.0035 \mathrm{e} / \AA$. The positive (negative) value indicates excess (defect) of induced electrons plotted in yellow (blue). Goldish, light grey, dark grey, and violet spheres indicate $\mathrm{Au}, \mathrm{H}, \mathrm{C}$, and $\mathrm{N}$ atoms, respectively. The interaction between the molecule and the gold surface (after due atomic relaxation) leads to a transfer of approximately 0.14 electrons from the molecule to the substrate following our Bader charge analysis. 


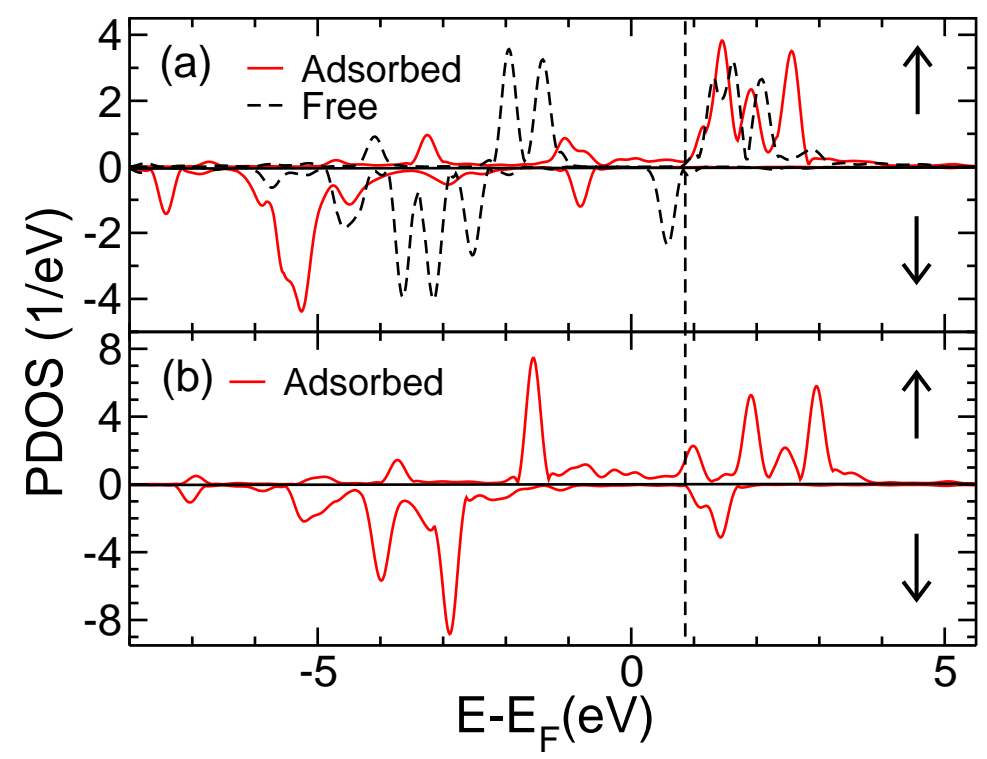

Figure 5: Density of states projected (PDOS) on the Fe $d$-manifold for $(a)$ the high-spin configuration $\left(4.3 \mu_{B}\right)$ and $(b)$ the low-spin one $\left(2.6 \mu_{B}\right)$. The black curve in $(a)$ corresponds to the free-molecule configuration with a magnetic moment of $2.0 \mu_{B}(\mathrm{~S}=1)$. The adsorbed high-spin configuration (red in $(a)$ ) corresponds to a full $d$-shell for the majority spin $(\downarrow)$ and approximately only one electron in the minority spin ( $\uparrow$ ) leading to 4 unpaired spins or a total spin $S=2$. This is in contrast with the free case (black) that displays two electrons from the minority spin but only four electrons for the majority spin, leading to a total spin $\mathrm{S}=1$. The low-spin configuration is closer to the free-molecule configuration although it displays some energy shifts similar to the high-spin one leading to a smaller occupation of the minority spin. As a consequence the low-spin configuration approximately corresponds to a total spin $S=1$. 
(a)

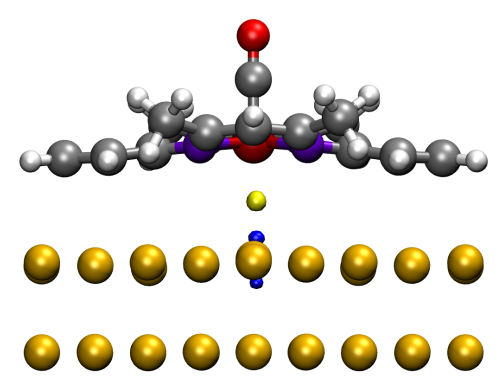

(b)

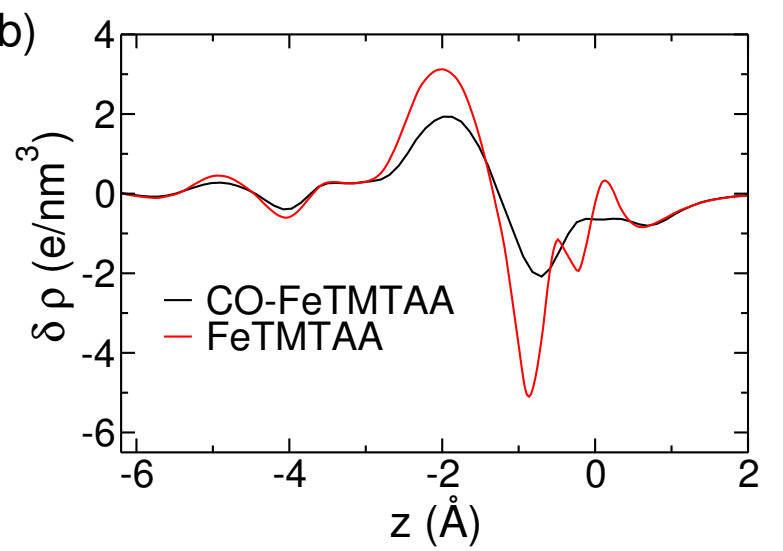

Figure 6: (a) Induced density between a CO-FeTMTAA molecule and the $\mathrm{Au}(111)$ substrate. Goldish, light grey, dark grey, violet, dark red and light red spheres indicate Au, H, C, N, $\mathrm{Fe}$, and $\mathrm{O}$ atoms, respectively. The isosurfaces are taken for the same values as in Fig. 4, showing the large difference in charge transfer between molecule and substrate depending on adding an extra carbon monoxide molecule. (b) Planar average of the induced charge for the CO-FeTMTAA complex (black) and the FeTMTAA molecule (red). The Fe atom of the molecule is the origin of coordinates. Positive values of $z$ correspond to the vacuum side. This graph reveals the surface dipole of the rearranged charges due to the interaction between molecular complex and substrate. At the isovalue of $(a)$, the planar average shows zero induced density for the CO-FeTMTAA molecule, in agreement with the very localized distribution of $(a)$. 


\section{References}

(1) Scheidt, W. R.; Reed, C. A. Chem. Rev. 1981, 81, 543-555.

(2) Goulle, V.; Harriman, A.; Lehn, J.-M. J. Chem. Soc., Chem. Commun., 1993, 10341036.

(3) Jung, O.-S.; Jo, D. H.; Lee, Y.-A.; Conklin, B. J.; Pierpont, C. G. Inorg. Chem. 1997, $36,19-24$.

(4) Powell, C. E.; Cifuentes, M. P.; Morrall, J. P.; Stranger, R.; Humphrey, M. G.; Samoc, M.; Luther-Davies, B.; Heath, G. A. J. Am. Chem. Soc. 2003, 125, 602-610.

(5) Tanaka, K.; Kitamura, N.; Takahashi, Y.; Chujo, Y. Bioorgan. Med. Chem. 2009, 17, $3818-3823$.

(6) Leoni, T.; Guillermet, O.; Walch, H.; Langlais, V.; Scheuermann, A.; Bonvoisin, J.; Gauthier, S. Phys. Rev. Lett. 2011, 106, 216103.

(7) Scherlis, D. A.; Cococcioni, M.; Sit, P.; Marzari, N. J. Phys. Chem. B 2007, 111, 7384-7391.

(8) Stróżecka, A.; Soriano, M.; Pascual, J. I.; Palacios, J. J. Phys. Rev. Lett. 2012, 109, 147202.

(9) Li Huang, Y.; Lu, Y.; Niu, T. C.; Huang, H.; Kera, S.; Ueno, N.; Wee, A. T. S.; Chen, W. Small 2012, 8, 1423-1428.

(10) Sedona, F.; Di Marino, M.; Forrer, D.; Vittadini, A.; Casarin, M.; Cossaro, A.; Floreano, L.; Verdini, A.; Sambi, M. Nat. Mater. 2012, 11, 970-977.

(11) Nilson, K.; Åhlund, J.; Shariati, M.-N.; Göthelid, E.; Palmgren, P.; Schiessling, J.; Berner, S.; Mårtensson, N.; Puglia, C. J. Phys. Chem. C 2010, 114, 12166-12172. 
(12) Bai, Y.; Buchner, F.; Wendahl, M. T.; Kellner, I.; Bayer, A.; Steinrück, H.-P.; Marbach, H.; Gottfried, J. M. J. Phys. Chem. C 2008, 112, 6087-6092.

(13) Ge, X.; Manzano, C.; Berndt, R.; Anger, L. T.; Köhler, F.; Herges, R. J. Am. Chem. Soc. 2009, 131, 6096-6098.

(14) Isvoranu, C.; Wang, B.; Schulte, K.; Ataman, E.; Knudsen, J.; Andersen, J. N.; Bocquet, M. L.; Schnadt, J. J. Phys.: Condens. Matter 2010, 22, 472002.

(15) Wäckerlin, C.; Chylarecka, D.; Kleibert, A.; Müller, K.; Iacovita, C.; Nolting, F.; Jung, T. A.; Ballav, N. Nat. Commun. 2010, 1, 1-7.

(16) Venkataramani, S.; Jana, U.; Dommaschk, M.; Sönnichsen, F. D.; Tuczek, F.; Herges, R. Science 2011, 331, 445-448.

(17) Seufert, K.; Auwärter, W.; Barth, J. V. J. Am. Chem. Soc. 2010, 132, 18141-18146.

(18) Hieringer, W.; Flechtner, K.; Kretschmann, A.; Seufert, K.; Auwärter, W.; Barth, J. V.; Görling, A.; Steinrück, H.-P.; Gottfried, J. M. J. Am. Chem. Soc. 2011, 133, 6206-6222.

(19) Seufert, K.; Bocquet, M.-L.; Auwärter, W.; Weber-Bargioni, A.; Reichert, J.; Lorente, N.; Barth, J. V. Nat. Chem. 2011, 3, 114-119.

(20) Gopakumar, T. G.; Tang, H.; Morillo, J.; Berndt, R. J. Am. Chem. Soc. 2012, 134 , $11844-11847$.

(21) Tanakaa, T.; Osuka, A. Chem. Soc. Rev. 2015, 44, 943-969.

(22) Wang, Y. F.; Wu, K.; Kröger, J.; Berndt, R. AIP Adv. 2012, 2, 041402.

(23) Ballav, N.; Wäckerlin, C.; Siewert, D.; Oppeneer, P. M.; Jung, T. A. J. Phys. Chem. Lett. 2013, 4, $2303-2311$.

(24) Gottfried, J. M. Surf. Sci. Rep. 2015, 70, $259-379$. 
(25) Auwärter, W.; Écija, D.; Klappenberger, F.; Barth, J. V. Nat. Chem. 2015, 7, 105-120.

(26) Hipps, K. W.; Scudiero, L.; Barlow, D. E.; Cooke, M. P. J. Am. Chem. Soc. 2002, 124, $2126-2127$.

(27) de Wild, M.; Berner, S.; Suzuki, H.; Yanagi, H.; Schlettwein, D.; Ivan, S.; Baratoff, A.; Güntherodt, H.-J.; Jung, T. A. ChemPhysChem 2002, 3, 881-885.

(28) Bonifazi, D.; Spillmann, H.; Kiebele, A.; de Wild, M.; Seiler, P.; Cheng, F.; Güntherodt, H.-J.; Jung, T.; Diederich, F. Angew. Chem. Int. Ed. 2004, 43, 47594763.

(29) Yang, Z.-Y.; Lei, S.-B.; Gan, L.-H.; Wan, L.-J.; Wang, C.; Bai, C.-L. ChemPhysChem 2005, 6, 65-70.

(30) Huang, Y. L.; Chen, W.; Wee, A. T. S. J. Am. Chem. Soc. 2011, 133, 820-825.

(31) Buchner, F.; Seufert, K.; Auwärter, W.; Heim, D.; Barth, J. V.; Flechtner, K.; Gottfried, J. M.; Steinrück, H.-P.; Marbach, H. ACS Nano 2009, 3, 1789-1794.

(32) Gopakumar, T. G.; Matino, F.; Schwager, B.; Bannwarth, A.; Tuczek, F.; Berndt, R. J. Phys. Chem. C 2010, 114, 18247-18251.

(33) Woodruff, W. H.; Pastor, R. W.; Dabrowiak, J. C. J. Am. Chem. Soc. 1976, 98, 79998006.

(34) Kresse, G.; Furthmüller, J. Comput. Mater. Sci. 1996, 6, 15-50.

(35) Perdew, J. P.; Burke, K.; Ernzerhof, M. Phys. Rev. Lett. 1996, 77, 3865-3868.

(36) Tkatchenko, A.; Scheffler, M. Phys. Rev. Lett. 2009, 102, 073005.

(37) Kresse, G.; Joubert, D. Phys. Rev. B 1999, 59, 1758-1775. 
(38) Dudarev, S. L.; Botton, G. A.; Savrasov, S. Y.; Humphreys, C. J.; Sutton, A. P. Phys. Rev. B 1998, 5\%, 1505-1509.

(39) Weiss, M. C.; Bursten, B.; Peng, S.-M.; Goedken, V. L. J. Am. Chem. Soc. 1976, 98, 8021-8031.

(40) We define $1 \mathrm{ML}$ as a coverage of 1 molecule per $\mathrm{nm}^{2}$, the most dense structure we observed (cf. Supporting Information).

(41) Harten, U.; Lahee, A. M.; Toennies, J. P.; Wöll, C. Phys. Rev. Lett. 1985, 54, 26192622.

(42) Wöll, C.; Chiang, S.; Wilson, R. J.; Lippel, P. H. Phys. Rev. B 1989, 39, 7988-7991.

(43) Borgogno, A.; Rastrelli, F.; Bagno, A. Dalton Trans. 2014, 43, 9486-9496.

(44) Gottfried, J.; Schmidt, K. J.; Schroeder, S. L. M.; Christmann, K. Surf. Sci. 2003, 536, 206-224.

(45) Charra, F.; Cousty, J. Phys. Rev. Lett. 1998, 80, 1682-1685.

(46) Böhringer, M.; Morgenstern, K.; W.-D. Schneider,; Berndt, R.; Mauri, F.; A. de Vita,; Car, R. Phys. Rev. Lett. 1999, 83, 324-327.

(47) Plass, K. E.; Grzesiak, A. L.; Matzger, A. J. Acc. Chem. Res. 2007, 40, 287-293.

(48) Ernst, K.-H. Phys. Status Solidi B 2012, 249, 2057-2088.

(49) Pawin, G.; Wong, K. L.; Kwon, K.-Y.; Bartels, L. Science 2006, 313, 961-962.

(50) Goedken, V. L.; Peng, S.-M.; Molin-Norris, J. A.; Park, Y.-a. J. Am. Chem. Soc. 1976, 98, 8391-8400.

(51) Klose, A.; Hesschenbrouck, J.; Solari, E.; Latronico, M.; Floriani, C.; Re, N.; ChiesiVilla, A.; Rizzoli, C. J. Organomet. Chem. 1999, 591, 45-62. 
(52) Silva, A. L. P.; de Almeida, L. F.; Marques, A. L. B.; Varela, J. d. J. G.; Tanaka, A. A.; da Silva, A. B. F. Polyhedron 2014, 67, 36-43.

(53) Bartels, L.; Meyer, G.; Rieder, K.-H. Appl. Phys. Lett. 1997, 71, 213-215.

(54) Tiwari, R. K.; Otálvaro, D. M.; Joachim, C.; Saeys, M. Surf. Sci. 2009, 603, 3286-3291.

(55) Liu, L.; Yang, K.; Jiang, Y.; Song, B.; Xiao, W.; Li, L.; Zhou, H.; Wang, Y.; Du, S.; Ouyang, M.; Hofer, W. A.; Castro Neto, A. H.; Gao, H.-J. Sci. Rep. 2013, 3, 1210.

(56) Knorr, N.; Brune, H.; Epple, M.; Hirstein, A.; Schneider, M. A.; Kern, K. Phys. Rev. B 2002, 65, 115420 .

(57) Repp, J.; Moresco, F.; Meyer, G.; Rieder, K.-H.; Hyldgaard, P.; Persson, M. Phys. Rev. Lett. 2000, 85, 2981-2984.

(58) Lukas, S.; Witte, G.; Wöll, C. Phys. Rev. Lett. 2001, 88, 028301.

(59) Silly, F.; Pivetta, M.; Ternes, M.; Patthey, F.; Pelz, J. P.; Schneider, W. D. Phys. Rev. Lett. 2004, 92, 016101.

(60) Wang, Y.; Ge, X.; Manzano, C.; Kröger, J.; Berndt, R.; Hofer, W. A.; Tang, H.; Cerda, J. J. Am. Chem. Soc. 2009, 131, 10400-10402.

(61) Yokoyama, T.; Takahashi, T.; Shinozaki, K.; Okamoto, M. Phys. Rev. Lett. 2007, 98, 206102.

(62) Cheng, Z.; Gao, L.; Deng, Z.; Jiang, N.; Liu, Q.; Shi, D.; Du, S.; Guo, H.; Gao, H.-J. J. Phys. Chem. C 2007, 111, 9240-9244.

(63) Fernandez-Torrente, I.; Monturet, S.; Franke, K.; Fraxedas, J.; Lorente, N.; Pascual, J. Phys. Rev. Lett. 2007, 99, 176103. 
(64) Fraxedas, J.; García-Gil, S.; Monturet, S.; Lorente, N.; Fernández-Torrente, I.; Franke, K. J.; Pascual, J. I.; Vollmer, A.; Blum, R.-P.; Koch, N.; Ordejón, P. J. Phys. Chem. C 2011, 115, 18640-18648.

(65) Gopakumar, T. G.; Brumme, T.; Kröger, J.; Toher, C.; Cuniberti, G.; Berndt, R. J. Phys. Chem. C 2011, 115, 12173-12179.

(66) Ziegler, M.; Néel, N.; Sperl, A.; Kröger, J.; Berndt, R. Phys. Rev. B 2009, 80, 125402. 